\title{
Searching for optimal positions through directional data in a political competition model
}

\section{Ma Dolores López*}

Departamento de Matemática e Informática Aplicadas

a la Ingeniería Civil de la E.T.S.I. Caminos,

Canales y Puertos,

Universidad Politécnica de Madrid, Spain

Email: marilo.lopez@upm.es

*Corresponding author

\section{Javier Rodrigo}

Departamento de Matemática Aplicada,

E.T.S. de Ingeniería,

Universidad Pontificia Comillas de Madrid, Spain

Email: jrodrigo@upcomillas.es

\section{Sagrario Lantarón}

Departamento de Matemática e Informática Aplicadas a la Ingeniería Civil de la E.T.S.I. Caminos,

Canales y Puertos,

Universidad Politécnica de Madrid, Spain

Email: sagrario.lantaron@upm.es

\begin{abstract}
This paper considers an application of directional data to political science. A model is presented in which the political preferences of the type of voters of a population are represented as points of the circumference unit with the political parties searching for optimal positions on this in order to gain the most support of that finite set of voter types. Strategies have been developed to search for optimal positions in the case of one party and Nash equilibrium positions are found in the game with two parties. The results reveal how the different parties redirect their stances to adapt to changing situations generated by economic or social circumstances affecting citizen preferences.
\end{abstract}

Keywords: political competition; game theory; Nash equilibrium; applications of OR techniques; geometric programming; exact algorithms; optimisation; OR models. 
Biographical notes: $M^{a}$ Dolores López has always been related with the university and currently she is a Professor at the Polytechnic University of Madrid where she joined the research group Applied Mathematic to the Civil Engineering. Her research interests include: game theory, applied mathematics to the political theory, localisation and computational geometry. She has published paper in journals of OR and game theory such as EJOR or IJGT.

Javier Rodrigo studied Mathematics at the Autónoma University, Madrid, Spain. He has always been related with the university and currently he is a Professor at the Universidad Pontificia Comillas (Madrid). He joined the research group Applied Mathematics to the Civil Engineering. His research interests include: game theory, discrete mathematics, applied mathematics to the political theory, localisation and computational geometry. He has published paper in journals of OR and game theory such as EJOR or IJGT.

Sagrario Lantarón studied Industrial Engineering at Santander, Spain. She has always been related with the University and currently she is a Professor at the Polytechnic University of Madrid where she joined the research group Applied Mathematics to the Civil Engineering. Her research interests include programming languages and computational science. She has published papers in journals related to computer science.

\section{Introduction}

Directional data appear naturally in various areas and are especially common in biological, meteorological and ecological sciences. Some applications are found, for example, in the analysis of wind directions, bird migration directions, geological deposits orientation, axial data analysis, allocation, etc. A detailed review of this matter may be found in Fisher et al. (1987), Fisher (1993), Mardia (1972) and more recently in Mardia and Jupp (2000), Jammalamadaka and Lund (2006), Arnold and SenGupta (2006), Qin et al. (2010) or Biswas et al. (2014). Directional data are usually treated with statistical techniques; see for example El-Hadidy (2015) where the circular normal distribution is applied.

In this paper, we develop an application of the study and treatment of directional data, specifically circular data, to political science from a game theory viewpoint. An initial introduction to the analysis of the application of circular data to political science may be found in Hangartner and Gill (2010). The decision to use circular data in this work is justified by the fact that political stances of a population of voters may be modelled through a segment whose centre would represent the most moderate stance while the extremes present the most radical stances to the right and left of said central stance. However, the most radical stances (right-wing extreme and left-wing extreme) actually have a common basis of thought and common lines of action based on certain violent methods. Thus, identifying the two extremes of the segment in order to generate a circumference may be considered a suitable starting point to define a political space (Hinich and Pollard, 1981).

Therefore a circumference centred on the origin of coordinates and radius unit is considered to be the one dimensional work space. It shall be assumed that the most moderate central stance occupies the east pole of said circumference and the west pole shall be the point that identifies the radical ideologies. 
Political competition models developed in the plane in which equilibrium positions are sought (Nash, 1951) establish that in situations of economic and social stability, such balance, if fact existing, is unique and in a central position with respect to voters' preferences (see Plott, 1967; Kramer, 1973; McKelvey, 1976). Thus political parties must present similar moderate stances in order to achieve the greatest number of voters (Abellanas et al., 2010, 2011; Roemer, 2001; López and Rodrigo, 2009).

However, in times of crisis and social tension, a radicalisation phenomenon appears in both the population and in the political decisions that the political parties must make. This means a polarisation of ideologies in concentrated groups of more extremist tendencies. Therefore, it may be of interest, from the party's decision-making point of view, to respond to certain citizen problems, find the political stance that said party must choose in order to approach the greatest number of voters in this new scenario, and adapt its program to said political stance. This new stance shall no longer be the central option that is produced in the previously described models.

With this in mind this paper considers a new deterministic geometric type of study in which optimal positions (political stances or decisions) are precisely determined, so as to be used by parties to attract the maximum number of voters in the scenario described above, through location and computational geometry techniques (Abellanas et al., 2006; Díaz-Báñez et al., 2011; Bhattacharya and Nandy, 2013). We also find Nash equilibrium solutions in the case of two competing parties. These results could be relevant for the party leaders in order to design the strategies of their political parties to face electoral processes.

The article is structured as follows: in Section 2, the model for the single party case is formalised by examining its optimal positions; Section 3 considers the model for two competing parties, examining the Nash equilibrium positions. Finally, the conclusions of the article are presented as well as possible extensions and future lines of work.

\section{The single party case}

\subsection{Formalisation of the model}

Following the model described in the previous section, all voters' political preferences of one population are given by a finite set of types $H=\left\{p_{1}, \ldots, p_{n}\right\}$ (Roemer, 2001). These types are represented as points of the circumference unit $C$ given by their polar coordinates $\left(1, \theta_{i}\right) i=1 \ldots n\left(-\pi<\theta_{i} \leq \pi\right)$. For simplification purposes, we shall now identify the points by their argument $\theta_{i}$.

As stated in the introduction, the angle $\theta=0$ represents the most moderate position or politic and $\theta=\pi$ represents the merging point of the radical stances to the right or to the left, therefore farther from the central stance.

Positive angles represent a more right-wing stance with respect to the moderate central position while negative angles represent the more left-wing stances.

In order to adapt the model to the political reality, it is assumed that the types of voters $p_{i}$ are not equally distributed. Thus it appears reasonable to consider a distribution of weights for the types of voters.

Each position's weight $p_{i}$ is then defined as $\operatorname{weight}\left(p_{i}\right)=k_{i}$ being $k_{1}+\ldots+k_{n}=n$. The weight of a subset of types shall be the sum of the weights of the types contained in it (Lillo et al., 2007). 
A political stance $\theta$ adopted by the party shall be considered to win the types of voters $\theta_{i}$ who are at a distance from such stance that is lower or equal to a particular boundary value $\varphi_{i}$ with $\varphi_{i} \geq 0$. Said boundary value represents the level or radius of tolerance allowed by the types of voters in order to vote for a party that offers the policy $\theta$. The closed circumference arc of length $2 \varphi_{i}$, whose polar extremes are the points $\theta_{i}-\varphi_{i}, \theta_{i}+\varphi_{i}$ shall be named the arc of tolerance for the type $\theta_{i}$ and shall be represented as $\left[\theta_{i}-\varphi_{i}, \theta_{i}+\varphi_{i}\right]$ (see Figure 1$)$.

Figure 1 Positions of types of voters (in radians) with their levels of tolerance (see online version for colours)

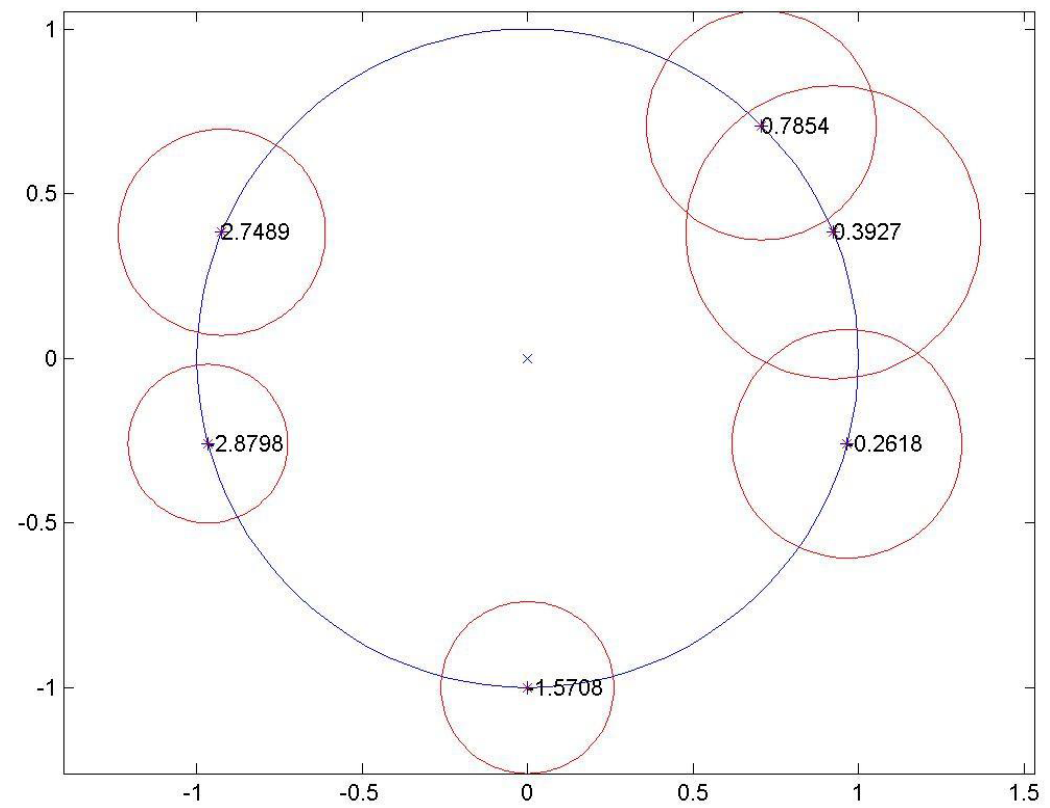

The considered distance, $d\left(\theta, \theta_{i}\right)$, shall be defined in the circumference as the length of the smallest arc that separates said points.

Thus, the party shall be interested in finding the optimal positions within the circumference unit that maximise the weight of the set of voters types, providing that the party is contained within their arcs of tolerance.

\subsection{Search of optimal positions}

The following methodology is used to find the aforementioned optimal positions: search for the intersection area of the arcs of tolerance with maximum weight. This area shall constitute the optimal region for the party to be situated.

Before searching for the optimal positions of the party in the political space, some assumptions regarding the previously defined $\varphi_{i}$ shall be offered in order to fit the political reality:

It is assumed that $\varphi_{i}$ diminishes as $\theta_{i}$ approaches $-\pi$ or $\pi$. This reflects the fact that extreme stances are more intransigent and therefore have a low level of tolerance.

In addition, it shall be assumed that 


$$
\left\{\begin{array}{ll}
\varphi_{i}<d\left(\theta_{i}, \pi\right) & \text { if } \theta_{i} \neq \pi \\
\varphi_{i}=0 & \text { if } \theta_{i}=\pi
\end{array} .\right.
$$

This ensures that any position chosen by the party cannot simultaneously gain support from the right-wing extreme and the left-wing extreme, despite their closeness to one another in the circumference.

Next, it is necessary to search, as the optimal region for the party, the intersection area of the arcs of circumference whose extremes are $\theta_{i}-\varphi_{i}, \theta_{i}+\varphi_{i}, i=1, \ldots, n$ which gives maximum weight. The sum of the weights of the voters corresponding to the arcs that intersect in that area, shall be the greatest potential winning that the party can achieve.

\subsubsection{Statement of the algorithm}

Below we present an exact algorithm that calculates the area of maximum winning for the voter types. It is based on the algorithms that search for maximum overlapping regions (see Cormen, 2009).

1 Input:

- $\quad$ The $n$ positions of the types of voters $\theta_{1}, \ldots, \theta_{n}$ within the circumference unit and their respective weights $k_{i}, i=1, \ldots, n$.

- $\quad$ The levels of tolerance $\varphi_{i}, i=1, \ldots, n$.

2 Output:

- The optimal positions for the party within the circumference unit and the winning in such positions.

Previous step: Verify that the radii of tolerance fulfil the model conditions.

Step 1 Consider the list $L$ formed by the extremes of the arcs of circumference centred in each type of voter $\theta_{i}$ and radius its level of tolerance: $\left\{x_{i}, x_{i}^{\prime}\right\}$. Sort said list by the angle of positioning of its points. The variable $x$ is assigned the first of the points of the list $L: x=x_{1}$. Observation: If points $x_{i}$ and $x_{i}^{\prime}$ coincide because the level of tolerance is 0 (which occurs in $\theta_{i}=\pi$ ), $x_{i}$ shall be considered previous to $x_{i}^{\prime}$ in the list $L$.

Step 2 Two counters $m$ and $c$ are initiated as 0 . Go through the list $L$ doing the following operations in each element:

- if it is an element of the form $x_{i}$, it increases the counter $c$ in $k_{i}$

- if it is an element of the form $x_{i}^{\prime}$, it decreases the counter $c$ in $k_{i}$.

When $c>m, m$ is redefined as $c$ and $x$ as $x_{i}$.

When $c=m x_{i}$ is saved in a new variable more to take into account the case where there is more than one arc with maximum weight.

When the process finishes, the counter indicates the maximum weight that the party may achieve. The arcs where it must be placed for this shall be those having $x$ and the next point of the list $L$ as extremes or, when more is non-empty, the arcs whose extreme points are those stored in more and the next points of the list $L$. 


\section{The case of two parties}

Despite the study of strategies considering just one party, in real world there are usually two major parties that compete for the voters. Therefore, a variation of the problem has been proposed, based on a political competition game in which two parties $p$ and $q$ are located on the unit circumference $C$ to attract the greatest weight possible of a finite set $H$ of points of $C$ (the voters). Nash (1951) equilibrium positions are examined in this new model.

\subsection{Formalisation of the model}

If each point of $H$ is represented by its angle in $C, \theta_{i}$ and has an arc of tolerance $\left[\theta_{i}-\varphi_{i}, \theta_{i}+\varphi_{i}\right]$ and $p$ and $q$ are located in positions with respective angles $\theta^{\prime}, \theta^{\prime \prime}, p$ will gain the weight of the points $p_{i}$ such that $\theta^{\prime} \in\left[\theta_{i}-\varphi_{i}, \theta_{i}+\varphi_{i}\right]$ and $d\left(\theta^{\prime}, \theta_{i}\right)<d\left(\theta^{\prime \prime}, \theta_{i}\right)$, whereas $q$ will gain the weight of the points $p_{i}$ such that $\theta^{\prime \prime} \in\left[\theta_{i}-\varphi_{i}, \theta_{i}+\varphi_{i}\right]$ and $d\left(\theta^{\prime \prime}, \theta_{i}\right)<d\left(\theta^{\prime}, \theta_{i}\right)$, distributing half of the weight of the $p_{i}$ such that $\theta^{\prime}, \theta^{\prime \prime} \in\left[\theta_{i}-\varphi_{i}, \theta_{i}+\varphi_{i}\right]$ and $d\left(\theta, \theta_{i}\right)=d\left(\theta^{\prime}, \theta_{i}\right)$.

In a more formal way, if we define:

$$
\begin{gathered}
\chi_{i}^{1}\left(\theta^{\prime}, \theta^{\prime \prime}\right)= \begin{cases}k_{i} & \text { if } \theta^{\prime} \in\left[\theta_{i}-\varphi_{i}, \theta_{i}+\varphi_{i}\right], d\left(\theta^{\prime}, \theta_{i}\right)<d\left(\theta^{\prime \prime}, \theta_{i}\right) \\
0 & \text { if } \theta^{\prime} \notin\left[\theta_{i}-\varphi_{i}, \theta_{i}+\varphi_{i}\right] \text { or } d\left(\theta^{\prime}, \theta_{i}\right)>d\left(\theta^{\prime \prime}, \theta_{i}\right) \\
\frac{k_{i}}{2} & \text { if } \theta^{\prime} \in\left[\theta_{i}-\varphi_{i}, \theta_{i}+\varphi_{i}\right], d\left(\theta^{\prime}, \theta_{i}\right)=d\left(\theta^{\prime \prime}, \theta_{i}\right)\end{cases} \\
\chi_{i}^{2}\left(\theta^{\prime}, \theta^{\prime \prime}\right)= \begin{cases}k_{i} & \text { if } \theta^{\prime \prime} \in\left[\theta_{i}-\varphi_{i}, \theta_{i}+\varphi_{i}\right], d\left(\theta^{\prime \prime}, \theta_{i}\right)<d\left(\theta^{\prime}, \theta_{i}\right) \\
0 & \text { if } \theta^{\prime \prime} \notin\left[\theta_{i}-\varphi_{i}, \theta_{i}+\varphi_{i}\right] \text { or } d\left(\theta^{\prime \prime}, \theta_{i}\right)>d\left(\theta^{\prime}, \theta_{i}\right) \\
\frac{k_{i}}{2} & \text { if } \theta^{\prime \prime} \in\left[\theta_{i}-\varphi_{i}, \theta_{i}+\varphi_{i}\right], d\left(\theta^{\prime \prime}, \theta_{i}\right)=d\left(\theta^{\prime}, \theta_{i}\right)\end{cases}
\end{gathered}
$$

So, if they are positioned in $\theta^{\prime}, \theta^{\prime \prime}$ the payoff functions of the parties $p$ and $q$ are respectively:

$$
\Pi^{1}\left(\theta^{\prime}, \theta^{\prime \prime}\right)=\sum_{i=1}^{n} \chi_{i}^{1}\left(\theta^{\prime}, \theta^{\prime \prime}\right), \Pi^{2}\left(\theta^{\prime}, \theta^{\prime \prime}\right)=\sum_{i=1}^{n} \chi_{i}^{2}\left(\theta^{\prime}, \theta^{\prime \prime}\right) .
$$

Remark 1:

a Generally, the game is not of sum $n$ because there may be angles $\theta_{i}$ such that $\theta^{\prime}, \theta^{\prime \prime}$ do not belong to $\left[\theta_{i}-\varphi_{i}, \theta_{i}+\varphi_{i}\right]$, so $k_{i}$ is none of the two parties' winnings. Therefore von Neumann's conditions cannot be applied (Neumann and Morgenstern, 1944) to ensure the existence of Nash equilibrium since it is not a constant sum game. For the study of non-constant sum games, see Fox (2010).

b This model may be interpreted as a version with discrete payoffs of the Downs game (Roemer, 2001). 


\subsection{Equilibrium study}

The Nash equilibrium positions in the proposed game have been examined. Thus the following notations have been introduced.

- $\quad M$ is the greatest weight of the points of $H$ that a party may obtain if it is situated on $C$ without competing with the other party.

- $\quad R$ is the region in $C$ where $M$ is attained.

It must be observed that if we define

$$
\chi_{i}(\theta)= \begin{cases}k_{i} & \text { if } \theta \in\left[\theta_{i}-\varphi_{i}, \theta_{i}+\varphi_{i}\right] \\ 0 & \text { if } \theta \notin\left[\theta_{i}-\varphi_{i}, \theta_{i}+\varphi_{i}\right]\end{cases}
$$

it stands that $M=\max _{\theta \in C} \sum_{i=1}^{n} \chi_{i}(\theta)$. We also have that $\Pi^{i}\left(\theta^{\prime}, \theta^{\prime \prime}\right) \leq M, i=1,2$. There are the following sufficient conditions for existence of Nash equilibrium positions:

Proposition 3.1: If $R$ has more than one connected component and there are no intervals: $\left[\theta_{i}-\varphi_{i}, \theta_{i}+\varphi_{i}\right], i=1, \ldots, n$, such that $\left[\theta_{i}-\varphi_{i}, \theta_{i}+\varphi_{i}\right] \cap R_{j} \neq \phi$ and $\left[\theta_{i}-\varphi_{i}, \theta_{i}+\varphi_{i}\right] \cap R_{k} \neq \phi$ being $R_{j}, R_{k}$ two connected components of $R$, then any position $\left(\theta^{\prime}, \theta^{\prime \prime}\right)$ with $\theta^{\prime} \in R_{j}, \theta^{\prime \prime} \in R_{k}$ is of Nash equilibrium.

Proof: Those positions fulfil $\Pi^{1}\left(\theta^{\prime}, \theta^{\prime \prime}\right)=\Pi^{2}\left(\theta^{\prime}, \theta^{\prime \prime}\right)=M$ : if, for instance, $\left[\theta_{1}-\varphi_{1}, \theta_{1}+\varphi_{1}\right], \ldots,\left\lfloor\theta_{i_{0}}-\varphi_{i 0}, \theta_{i 0}+\varphi_{i 0}\right\rfloor$ are the intervals that intersect in $R_{j}$ and $\left[\theta_{i_{0+1}}-\varphi_{i_{0+1}}, \theta_{i_{0+1}}+\varphi_{i_{0+1}}\right], \ldots,\left\lfloor\theta_{i_{0}+l}-\varphi_{i_{0}+l}, \theta_{i_{0}+l}+\varphi_{i_{0}+l}\right\rfloor$ are the intervals that intersect in $R_{k}$, then we have that $\theta^{\prime} \in\left[\theta_{s}-\varphi_{s}, \theta_{s}+\varphi_{s}\right]$ for every $s=1, \ldots, i_{0}$ and $d\left(\theta^{\prime}, \theta_{s}\right)<d\left(\theta^{\prime \prime}, \theta_{s}\right)$. for every $s=1, \ldots, i_{0}$, since $d\left(\theta^{\prime}, \theta_{s}\right) \leq \varphi_{s}$ and $d\left(\theta^{\prime \prime}, \theta_{s}\right)>\varphi_{s}$ by the hypothesis. Therefore: $\Pi^{1}\left(\theta^{\prime}, \theta^{\prime \prime}\right)=k_{1}+\ldots+k_{i_{0}}=M$. In an analogous way we have that: $\Pi^{2}\left(\theta^{\prime}, \theta^{\prime \prime}\right)=k_{i_{0}+1}+\ldots+k_{l}=M$. Therefore it is a Nash equilibrium position since in it, the parties have the highest pay off possible. \#

\section{Remark 2:}

a So that there can be equilibrium positions as the ones referred to in Proposition 3.1, it must be $M \leq \frac{n}{2}$, since $2 M=\Pi^{1}\left(\theta^{\prime}, \theta^{\prime \prime}\right)+\Pi^{2}\left(\theta^{\prime}, \theta^{\prime \prime}\right) \leq n$.

b In the equilibrium positions of Proposition 3.1 each party wins a set of points for which it does not fight with the other party, because the latter is not in the arcs of tolerance of the points of such set. So Proposition 3.1 gives Nash equilibrium positions for which the two parties do not share location. Below is a generalisation of Proposition 3.1:

Proposition 3.2: If the connected components of $R$ are $R_{1}, \ldots, R_{s}$ and for every $j, k$ such that $1 \leq j, k \leq s, j \neq k$, there are no intervals $\left[\theta_{i}-\varphi_{i}, \theta_{i}+\varphi_{i}\right], i=1, \ldots, n$, such that $\left[\theta_{i}-\varphi_{i}\right.$, $\left.\theta_{i}+\varphi_{i}\right] \cap R_{j} \neq \phi$ and $\left[\theta_{i}-\varphi_{i}, \theta_{i}+\varphi_{i}\right] \cap R_{k} \neq \phi$, then every Nash equilibrium position in which $\theta^{\prime}$ or $\theta^{\prime \prime}$ belong to $R$ are the $\left(\theta^{\prime}, \theta^{\prime \prime}\right)$ with $\theta^{\prime} \in R_{j}, \theta^{\prime \prime} \in R_{k}$. 
Proof: The positions $\left(\theta^{\prime}, \theta^{\prime \prime}\right)$ with $\theta^{\prime} \in R_{j}, \theta^{\prime \prime} \in R_{k}, j \neq k$, are of Nash equilibrium as a result of Proposition 3.1. We see that they are the only ones:

If, for instance, $\theta^{\prime} \notin R_{j}$ and $\theta^{\prime \prime} \in R_{k}$, then $\Pi^{1}\left(\theta^{\prime}, \theta^{\prime \prime}\right)<M$, so the first party can move to a position $\theta \in R_{j}$ for $j$ such that $\theta^{\prime \prime} \notin R_{j}$ and obtain $\Pi^{1}\left(\theta^{\prime}, \theta^{\prime \prime}\right)=M$ as we saw in the proof of Proposition 3.1, so it increases its winnings and $\left(\theta^{\prime}, \theta^{\prime \prime}\right)$ is not of equilibrium. If, $\theta^{\prime}, \theta^{\prime \prime} \in R_{j} \quad$ for $\quad$ a certain $j \in\left\{\begin{array}{lll}1, & \ldots, & s\end{array}\right\}$, then $\Pi^{i}\left(\theta^{\prime}, \theta^{\prime \prime}\right)<M$ for a $i \in\{1,2\}$, since it is fulfilled that $\Pi^{1}\left(\theta^{\prime}, \theta^{\prime \prime}\right)+\Pi^{2}\left(\theta^{\prime}, \theta^{\prime \prime}\right)=M$. If, for instance, $\Pi^{1}\left(\theta^{\prime}, \theta^{\prime \prime}\right)<M$, the first party can move to a position $\theta \in R_{k}$ for $k \neq j$ and obtain $\Pi^{1}\left(\theta^{\prime}, \theta^{\prime \prime}\right)=M$, thus increasing its winnings and $\left(\theta^{\prime}, \theta^{\prime \prime}\right)$ is not of equilibrium. \#

Below are some necessary conditions for the existence of Nash equilibrium when $R$ is connected. First, there is a condition on the winnings.

Proposition 3.3: If $R$ is connected and $\left(\theta^{\prime}, \theta^{\prime \prime}\right)$ is a Nash equilibrium position with $\theta^{\prime}, \theta^{\prime \prime} \in R$, then $\Pi^{i}\left(\theta^{\prime}, \theta^{\prime \prime}\right)=\frac{M}{2}$ for $i=1,2$.

Proof: If, for instance, $\Pi^{1}\left(\theta^{\prime}, \theta^{\prime \prime}\right)=\frac{M}{2}$, then the first party can deviate to $\theta^{\prime \prime}$ in order to obtain $\Pi^{1}\left(\theta^{\prime \prime}, \theta^{\prime \prime}\right)=\frac{M}{2}$ and improve the winnings, contradicting the fact that $\left(\theta^{\prime}, \theta^{\prime \prime}\right)$ is a Nash equilibrium position.

If $\Pi^{1}\left(\theta^{\prime}, \theta^{\prime \prime}\right)>\frac{M}{2}$, then it will be $\Pi^{2}\left(\theta^{\prime}, \theta^{\prime \prime}\right)=M-\Pi^{1}\left(\theta^{\prime}, \theta^{\prime \prime}\right)<\frac{M}{2}$, then once again there is a contradiction.

Therefore $\Pi^{1}\left(\theta^{\prime}, \theta^{\prime \prime}\right)=\Pi^{2}\left(\theta^{\prime}, \theta^{\prime \prime}\right)=\frac{M}{2}$. \#

Remark 3: In the potential Nash equilibrium positions for this proposition, as well as in those obtained from Proposition 3.1, it is verified that $\Pi^{i}\left(\theta^{\prime}, \theta^{\prime \prime}\right) \leq \frac{n}{2}$ for $i=1,2$.

In order to provide a new necessary equilibrium condition, a preliminary definition must be given:

Definition 3.1: Assume that $R$ is connected, $\theta_{i_{1}}, \ldots ., \theta_{i_{k}} \in H$ and they fulfil $\left[\theta_{i_{1}}-\varphi_{i_{1}}, \theta_{i_{1}}+\varphi_{i_{1}}\right] \cap \ldots \cap\left[\theta_{i_{k}}-\varphi_{i_{k}}, \theta_{i_{k}}+\varphi_{i_{k}}\right]=R$. Consider the subsets of $\left\{\theta_{i_{1}}, \ldots, \theta_{i_{k}}\right\}$ such that their weights are greater than $\frac{M}{2}, H_{1}, \ldots, H_{l}$. For each $H_{i}, i=1, \ldots, l$, consider the arc in $C$ of a shortest length that contains $H_{i}$. We call $I_{\frac{M}{2}}$ the intersection of said arcs.

Proposition 3.4: If $R$ is connected and $\left(\theta^{\prime}, \theta^{\prime \prime}\right)$ is a Nash equilibrium position with $\theta^{\prime}, \theta^{\prime \prime} \in R$, then $\theta^{\prime}, \theta^{\prime \prime} \in I_{\frac{M}{2}}$. 
Proof: If, for instance, $\theta^{\prime \prime} \notin I_{\frac{M}{2}}$. in a position $\left(\theta^{\prime}, \theta^{\prime \prime}\right)$ fulfilling the conditions of the proposition, then there is a subset of $\left\{\theta_{i_{1}}, \ldots ., \theta_{i_{k}}\right\}$ with a weight exceeding $\frac{M}{2}$, say $H_{1}$, such that $\theta^{\prime \prime}$ does not belong to the arc of shortest length containing $H_{1}$. If this arc remains, for instance, to the right of $\theta^{\prime \prime}$, then the first party may deviate to a position $\theta \in R$ to the right of $\theta^{\prime \prime}$ separating $\theta^{\prime \prime}$ from the points of $H_{1}$, so $\Pi^{1}\left(\theta^{\prime}, \theta^{\prime \prime}\right)>\frac{M}{2}$ and this party improves the winnings by Proposition 3 , contradicting the fact that $\left(\theta^{\prime}, \theta^{\prime \prime}\right)$ is a Nash equilibrium position. \#

Remark 4: According to this proposition, if the parties seek a stable position in the region of maximum payoff then they should search for the set $I_{\frac{M}{2}}$. As this set could be not a single point, the Nash equilibrium positions found with the presented methodology are not unique.

\section{Conclusions}

The main contribution of this paper is its suggestion of the use of circular data in the area of political economy, particularly in matters related to political location. This treatment is justified based on the viewpoint of the ideologies and therefore, it has been possible to develop a model which adapts itself to the different political scenarios of our current society. This model has solved the problem of determining optimal political positions, within the circumference, from which a party may choose in order to ensure the greatest possible number of voter types.

Games with circular strategy spaces have been found in the literature, as in Zhao et al. (2008). In that paper a finite space of strategies in the circumference has been considered. The novelty of the model we have presented, among other things, lies in the consideration of an infinite space of strategies combined with a finite set of possible winnings.

The theoretical results obtained have been effectively implemented in an exact search algorithm. This algorithm may be used to simulate different situations of political reality. This procedure could have managerial implications since it may be applied by the parties in order to create political strategies.

Finally, in the case of competition between two parties, conditions that ensure Nash equilibrium positions have been found. An important contribution of this model is that some of the determined positions of equilibrium do not fulfil the requirement that the two parties must choose the same strategy. This is a problem that usually arises in competition models between two players and for which different solutions have typically been found: restricting the positions of the players, studying mixed-strategy Nash equilibrium, weakening the definition of equilibrium, studying uncovered sets or considering valence issues, among others (see, for example, Abellanas et al., 2006, 2010, 2011; Roemer, 2001; McKelvey, 1976; Stokes, 1963; Díaz-Báñez et al., 2011). The use of arcs of tolerance is a new solution to this problem, that has plausible interpretations in various areas aside from political competition. 
This study has been developed for a simplified two-party model. Its generalisation to a greater number of parties seems to be a complex task. Regardless, the two-party model usually fits the reality of most countries in which generally, there are two major parties that compete for voter support.

A future research direction could consider providing an exact algorithm that finds the Nash equilibrium positions in the two-party game. To achieve this, it would probably be likely to segment the circular political space, as done in Hawkins and Lombard (2015).

\section{References}

Abellanas, M., Lillo, I., López, M. and Rodrigo, J. (2006) 'Electoral strategies in a dynamical democratic system', European Journal of Operational Research, Vol. 175, No. 2, pp.870-878.

Abellanas, M., Lillo, I., López, M. and Rodrigo, J. (2011) 'Weak equilibrium in a spatial model', International Journal of Game Theory, Vol. 40, No. 3, pp.449-459.

Abellanas, M., López, M. and Rodrigo, J. (2010) 'Searching for equilibrium positions in a game of political competition with restrictions', European Journal of Operational Research, Vol. 201, No. 3, pp.892-896.

Arnold, B.C. and SenGupta, A. (2006) 'Recent advances in the analyses of directional data in ecological and environmental sciences', Environmental and Ecological Statistics, Vol. 13, No. 3, pp.253-256.

Bhattacharya, B.B. and Nandy, S.C. (2013) 'New variations of the maximum coverage facility location problem', European Journal of Operational Research, Vol. 224, No. 3, pp.477-485.

Biswas, A., Dutta, S., Laha, A.K. and Bakshi, P.K. (2015) 'Response-adaptive allocation for circular data', Journal of Biopharmaceutical Statistics, Vol. 25, No. 4, pp.830-842.

Cormen, T.H. (2009) Introduction to Algorithms, MIT Press, USA.

Díaz-Báñez, J.M., Heredia, M., Pelegrín, B., Pérez-Lantero, P. and Ventura, I. (2011) 'Finding all pure strategy Nash equilibria in a planar location game', European Journal of Operational Research, Vol. 214, No. 1, pp.91-98.

El-Hadidy, M.A. (2015) 'Optimal spiral search plan for a randomly located target in the plane', International journal of Operational Research, Vol. 22, No. 4, pp.454-465.

Fisher, N.I. (1993) Statistical Analysis of Circular Data, University Press, Cambridge.

Fisher, N.I., Lewis, T. and Embleton, B.J.J. (1987) Statistical Analysis of Spherical Data, University Press, Cambridge.

Fox, W.P. (2010) 'Teaching the applications of optimisation in game theory's zero sum and non-zero sum games', International Journal of Data Analysis Techniques and Strategies, Vol. 2, No. 3, pp.258-284.

Hangartner, D. and Gill, J. (2010) 'Circular data in political science and how to handle it', Political Analysis, Vol. 18, No. 3, pp.316-336.

Hawkins, D.M. and Lombard, F. (2015) 'Segmentation of circular data', Journal of Applied Statistics, Vol. 42, No. 1, pp.88-97.

Hinich, M. and Pollard, W. (1981) 'A new approach to the spatial theory of electoral competition', American Journal of Political Science, Vol. 25, No. 2, pp.323-341.

Jammalamadaka, S.R. and Lund, U.J. (2006) 'The effect of wind direction on ozone levels: a case study', Environmental and Ecological Statistics, Vol. 13, No. 3, pp.287-298.

Kramer G.H. (1973) 'On a class of equilibrium conditions for majority rule', Econometrica, Vol. 42, No. 2, pp.285-297.

Lillo, I., López, M. and Rodrigo, J. (2007) 'A geometric study of the Nash equilibrium in a weighted case’, Applied Mathematical Sciences, Vol. 55, No. 1, pp.2715-2725. 
López, M. and Rodrigo, J. (2009) 'Discrete models of political competition', in Haugen I.N. and Nilsen A.S. (Eds.): Game Theory: Strategies, Equilibria and Theorems, pp.161-182, Nova Science Publishers, New York.

Mardia, K.V. (1972) Statistics of Directional Data, Academic Press, New York.

Mardia, K.V. and Jupp, P.E. (2000) Directional Statistics, Wiley, Chichester.

McKelvey, R.D. (1976) 'Intransitivities in multidimensional voting models and implications for agenda control', Journal of Economic Theory, Vol. 12, No. 3, pp.472-482.

Nash, J. (1951) 'Non-cooperative games', The Annals of Mathematics, Vol. 54, No. 2, pp.286-295.

Neumann, J. and Morgenstern, O. (1944) Theory of Games and Economic Behavior, Princeton University Press, Princeton.

Plott C.R. (1967) 'A notion of equilibrium and its possibility under majority rule', American Economic Review, Vol. 57, No. 4, pp.787-806.

Qin, X., Zhanga, S.J. and Yan, D.X. (2010) 'A new circular distribution and its application to wind data', Journal of Mathematics Research, Vol. 2, No. 3, pp.12-17.

Roemer, J. (2001) Political Competition, Harvard University Press, Harvard.

Stokes, D E. (1963) 'Spatial models of party competitions', American Politic Science Review, Vol. 57, No. 2, pp.368-377.

Zhao, Y., Chen, W. and Teng, S. (2008) 'The isolation game: a game of distances', in ISAAC: Proceedings of the 19th International Symposium on Algorithms and Computation, Springer, Berlin, Germany, pp.148-159.

\section{Appendix}

\section{Practical implementation of the algorithm of the Section 2.2}

The proposed procedure is described by the following MATLAB-like pseudocode.

Input of data:

Positions of the $n$ voters: array $V$ of $n$ elements

Tolerance of the $n$ voters: array $f i$ of $n$ elements

Weights of the $n$ voters: array $k$ of $n$ elements

\section{Compute $n$}

Compute matrix $L$ :

Set $j=1$;

for $i=1: n$

if $(f i(i)>=$ pi-abs $(V(i)))$ exit; end finalisation of the program if the previous step is not fulfilled

$L(j, 1)=V(i)-f i(i) ; L(j, 2)=k(i) ; L(j, 3)=1$; we mark with 1 the points $x_{i}$

$L(j+1,1)=V(i)+f i(i) ; L(j+1,2)=k(i) ; L(j+1,3)=2$; we mark with 2 the points

$$
x_{i}^{\prime} j=j+2
$$

end

Sort the rows of the matrix $\mathrm{L}$ according to the values of its first column. We consider the direction of order counter-clockwise from $-\pi$ to $\pi$

Set $m=0, c=0, x=1, n p=j-1, j=0$

for $i=2: n p$ 


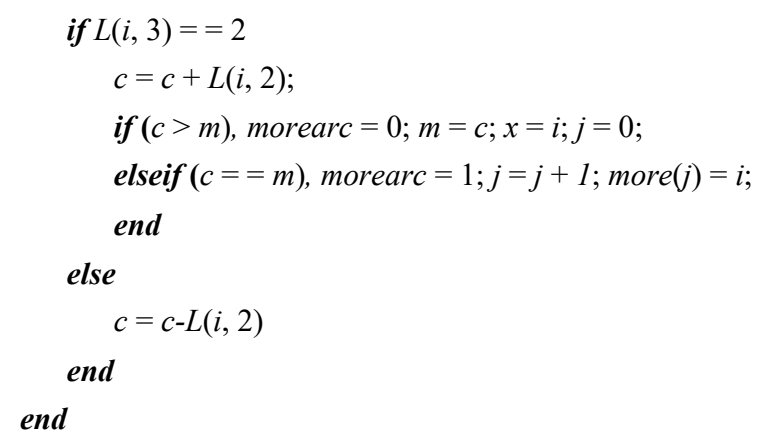

Solution arc $L(x, 1), L(x+1,1)$, maximum weight: $m$

If morearc there are more solution arcs with identical weight for $i=1: j$

solution additional $\operatorname{arc} L($ more $(i), 1), L($ more $(i)+1,1)$

end

end 